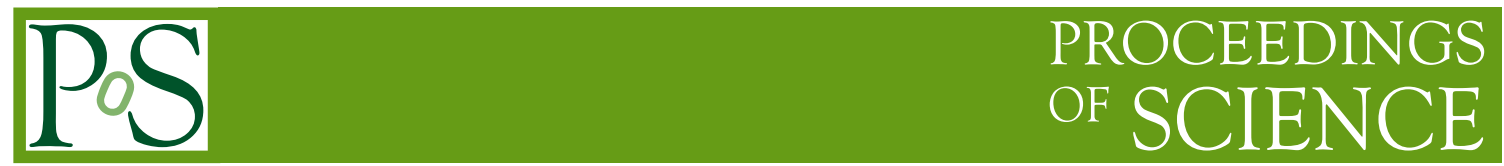

\title{
Local quartic interaction of scalars with higher spin gauge fields and commutator of linear gauge transformations
}

\author{
Ruben Manvelyan ${ }^{a, *}$ and Melik Karapetyan ${ }^{a}$ \\ ${ }^{a}$ Yerevan Physics Institute, \\ Alikhanian Br. Str. 2, 0036 Yerevan, Armenia \\ E-mail: manvel@yerphi.am, meliq.karapetyan@gmail.com
}

\begin{abstract}
Some special case of local quartic interaction of higher spin gauge field with a scalar field is considered. The nontrivial task of construction of interacting Lagrangian for the constrained higher spin field (physical gauge) was solved using the full power of Noether's procedure. As a byproduct linear on-field gauge transformation is obtained and the corresponding closure of commutator of transformation is analyzed. The right-hand side of this commutator is classified in respect to gauge transformations coming from cubic interactions of a scalar with different higher spin symmetric tensor fields and with mixed symmetry tensor field transformations.
\end{abstract}

RDP online workshop "Recent Advances in Mathematical Physics" - Regio2020,

5-6 December 2020

online

${ }^{*}$ Speaker 


\section{Motivation}

The problem of construction of complete Higher Spin (HS) Interaction Lagrangian is one of the tasks with smoldering interest for the last forty years [1]-[7]. From time to time this complicated and puzzling problem came to a relative center of interest when some even small progress was observed in interaction construction or when the HS theories start to play some role in the development of other important fields like AdS/CFT. Moreover, we can say that this task is interesting in itself due to the complexity and the necessity to develop and use nontrivial computing techniques even for small advances and achievements.

Even though during the last ten-twelve years we observed significant progress in the understanding of construction and structure of cubic interaction in different approaches, dimensions and backgrounds [8]-[20], our knowledge about quartic one is far from being complete and always bounded by the idea that this one should be nonlocal in general [21]-[26]. We should mention also that all these activities supplemented with the parallel development of Vasiliev's nonlinear and nonlagrangian theory of interacting HS field equations of motion in AdS background, where the questions of possible nonlocality beyond cubic level also arose and discussed. Nevertheless, it seems that in some special cases it is possible to construct some local quartic interactions between fields with different spins at least as a part of a more complicated covering theory (maybe nonlocal) including other gauge fields and symmetries.

In this paper, we construct some special local quartic interaction of two scalars and two spin four fields using standard Noether's procedure. The interesting points of this special construction are the following:

- First we see that to close Noether's procedure we should add additional cubic interaction of scalar with other spin gauge fields and corresponding HS gauge symmetries.

- Second important point that we constructing quartic vertex we derive fixed linear in gauge field gauge transformation of our HS field $\delta_{1}^{(\epsilon)}$ and then be able to investigate closure of commutators of two such a transformation

$$
\left[\delta_{1}^{(\eta)} \delta_{1}^{(\epsilon)}\right] \sim \delta_{1}^{(<<\eta, \epsilon>>)}+\text { additional terms }
$$

and understand whether it leads to nonlocality or not.

We organize our paper in the following way: in the next section, we show spin 2 exercise for construction similar quartic interaction in spin two cases. Then the essential Noether's construction is shown in section two with the final derivation of interaction Lagrangian and first order on HS gauge field gauge transformation. The last section is devoted to the investigation of the commutator of two $\delta_{1}$ transformations and classification of terms different from the same $\delta_{1}$ with the composed parameter on the right-hand side. These Notes are based on the long calculations which we performed using the technique and notation developed in the past in [27]-[32].

\section{Illustration: Spin two case}

For better understanding how we should construct special quartic interaction in higher spin case we start first from the following lagrangian for the interaction of scalar with spin 2 gauge field 
in the flat background:

$$
S^{\Phi \Phi h^{(2)}}=S_{0}(\Phi)+S_{1}\left(\Phi, h^{(2)}\right)
$$

where

$$
\begin{aligned}
S_{0}(\Phi) & =\frac{1}{2} \int d^{d} x \partial_{\mu} \Phi \partial^{\mu} \Phi \\
S_{1}\left(\Phi, h^{(2)}\right) & =\frac{1}{2} \int d^{d} x h^{(2) \mu \nu}\left[-\partial_{\mu} \Phi \partial_{\nu} \Phi+\frac{\eta_{\mu \nu}}{2} \partial_{\lambda} \Phi \partial^{\lambda} \Phi\right]
\end{aligned}
$$

This is a well known minimal coupling of scalar with gravity, linearized in flat background and the bracket in (3) is the usual energy-momentum tensor for massless scalar field. The spliting of (1) into the quadratic and cubic parts allows us to formulate the Noether's equations

$$
\delta_{1} S_{0}(\Phi)+\delta_{0} S_{1}\left(\Phi, h^{(2)}\right)=0
$$

where:

$$
\begin{aligned}
\delta_{0} h_{\mu \nu}^{(2)} & =\partial_{(\mu} \varepsilon_{\nu)}^{(1)}=\partial_{\mu} \varepsilon_{\nu}^{(1)}+\partial_{\nu} \varepsilon_{\mu}^{(1)} \\
\delta_{0} \Phi & =0 \\
\delta_{1} \Phi & =\varepsilon^{(1) \lambda} \partial_{\lambda} \Phi
\end{aligned}
$$

The crucial point here that we can discover interacting part (3) solving functional equation (4) variating known free part (2) in respect to admitting first order diffeomorphism of scalar (7) and using a zero-order variation of gauge field as an integration rule. Note also that the scalar field has no zero-order variation being matter field here.

Then we can formulate task for construction of the next order interaction of the two scalars with two spin two fields in the similar way:

$$
\delta_{2} S_{0}(\Phi)+\delta_{1} S_{1}\left(\Phi, h^{(2)}\right)+\delta_{0} S_{2}\left(\Phi, h^{(2)}\right)=0
$$

admitting that: $\delta_{2} \Phi=0$ we see that in this case we need to solve again two terms functional equation

$$
\delta_{1} S_{1}\left(\Phi, h^{(2)}\right)+\delta_{0} S_{2}\left(\Phi, h^{(2)}\right)=0
$$

using the same transformations (5)-(7) and introducing an assumption about the form of first order transformation of spin 2 gauge field:

$$
\delta_{1} h_{\mu \nu}^{(1)}=\varepsilon^{(1) \lambda} \partial_{\lambda} h_{\mu \nu}^{(2)}+\overline{\delta_{1}} h_{\mu \nu}^{(2)}
$$

where $\bar{\delta}_{1} h_{\mu \nu}$ we should find from equation (9) together with $S_{2}\left(\Phi, h^{(2)}\right)$. To show technology of solution we present variation of $S_{1}\left(\Phi, h^{(2)}\right)$ in the following form (after some algebra and partial integrations)

$$
\begin{aligned}
\delta_{1} S_{1}\left(\Phi, h^{(2)}\right) & =\int d^{d} x\left\{-\frac{1}{2} \partial^{\mu} \Phi \partial^{v} \Phi\left[\bar{\delta}_{1} h_{\mu \nu}^{(2)}-\partial_{(\mu} \varepsilon^{(1) \lambda} h_{\nu) \lambda}^{(2)}+2 h_{\mu}^{(2) \lambda} \partial_{(\nu} \varepsilon_{\lambda)}^{(1)}\right]\right. \\
& +\frac{1}{2} \partial^{\mu} \Phi \partial^{\nu} \Phi\left[\partial_{\lambda} \varepsilon^{(1) \lambda} h_{\mu \nu}^{(2)}+\frac{1}{2} \partial_{(\mu} \varepsilon_{\nu)}^{(1)} h_{\alpha}^{(2) \alpha}\right] \\
& \left.+\frac{1}{4} \partial^{\lambda} \Phi \partial_{\lambda} \Phi\left[\overline{\delta_{1}} h_{\alpha}^{(2) \alpha}-\partial_{\beta} \varepsilon^{(1) \beta} h_{\alpha}^{(2) \alpha}\right]\right\}
\end{aligned}
$$


From first line of (11) we can derive that if we define

$$
\overline{\delta_{1}} h_{\mu \nu}^{(2)}=\partial_{(\mu} \varepsilon^{(1) \lambda} h_{v) \lambda}^{(2)}
$$

then last term of first line can be integrated to $\delta_{0}\left[-\frac{1}{2} \partial^{\mu} \Phi \partial^{v} \Phi h_{\mu}^{\lambda} h_{\nu \lambda}\right]$

Then taking into account that

$$
\begin{aligned}
\bar{\delta}_{0} h_{\alpha}^{(2) \alpha} & =2 \partial_{\lambda} \varepsilon^{(1) \lambda} \\
\bar{\delta}_{1} h_{\alpha}^{(2) \alpha} & =2 \partial^{\lambda} \varepsilon^{(1) \alpha} h_{\lambda \alpha}^{(2)}=\frac{1}{2} \delta_{0}\left[h^{(2) \lambda \alpha} h_{\lambda \alpha}^{(2)}\right]
\end{aligned}
$$

we see that we can immediately integrate second and third line of (11) also and arrive to the following quartic action:

$$
\begin{aligned}
S_{2}\left(\Phi, h^{(2)}\right) & =\int d^{d} x\left\{\frac{1}{2} \partial^{\mu} \Phi \partial^{v} \Phi h_{\mu}^{(2) \lambda} h_{\nu \lambda}^{(2)}-\frac{1}{4} \partial^{\mu} \Phi \partial^{v} \Phi h_{\mu \nu}^{(2)} h_{\alpha}^{(2) \alpha}\right. \\
& \left.-\frac{1}{8} \partial^{\lambda} \Phi \partial_{\lambda} \Phi h^{(2) \alpha \beta} h_{\alpha \beta}^{(2)}+\frac{1}{16} \partial^{\lambda} \Phi \partial_{\lambda} \Phi h_{\alpha}^{(2) \alpha} h_{\beta}^{(2) \beta}\right\}
\end{aligned}
$$

with expected Lie derivative as a solution for first order variation of spin two fluctuation:

$$
\delta_{1}^{(\varepsilon)} h_{\mu \nu}^{(2)}=\varepsilon^{(1) \lambda} \partial_{\lambda} h_{\mu \nu}^{(2)}+\partial_{\mu} \varepsilon^{(1) \lambda} h_{\nu \lambda}^{(2)}+\partial_{\nu} \varepsilon^{(1) \lambda} h_{\mu \lambda}^{(2)}=\mathfrak{L}_{\varepsilon^{(1) \lambda}} h_{\mu \nu}^{(2)}
$$

with standard algebra

$$
\left[\delta_{1}^{(\eta)}, \delta_{1}^{(\varepsilon)}\right] h_{\mu \nu}^{(2)}=\delta_{1}^{(<<\varepsilon, \eta>>)} h_{\mu \nu}^{(2)}
$$

where composite parameter is the usual Lie commutator of vectors:

$$
<<\eta, \varepsilon>>=\left[\eta^{(1)}, \varepsilon^{(1)}\right]^{\lambda}=\eta^{(1) v} \partial_{\nu} \varepsilon^{(1) \lambda}-\varepsilon^{(1) v} \partial_{\nu} \eta^{(1) \lambda}
$$

Note that one can work with restricted external field also. As an example we can choose at once traceless $h^{(2) \mu v}$ supplemented with the corresponding constraint on gauge parameter $\partial_{\mu} \varepsilon^{(1) \mu}=0$. This means that in expression (11) survive only first line and first term of the last line. Then we see from (12) and (14) that $\bar{\delta}_{1} h_{\mu \nu}^{(2)}$ is not traceless but can be integrated. So we arrive to the first and third terms of interaction (15) describing interaction of $h^{(2) \mu \lambda} h_{\lambda}^{(2) v}$ with traceless part of current $J_{\mu \nu}^{(2)}=\partial_{\mu} \Phi \partial_{\nu} \Phi$.

\section{Spin four case}

setup

Our main task is to construct similar quartic interaction for spin 4 using prescriptions developed in the previous simple spin 2 case. In our previous articles [27], [28] we prove that in both $A d S$ and flat backgrounds after corresponding field redefinition interaction of even spin $s$ gauge field with spin $s$ current constructed from scalar and derivatives should be supplemented with the whole tower of invariant actions for couplings of the same scalar with all gauge fields of smaller even spin. So the starting lagrangian for our task we take from [28] rewriting all terms in the flat background:

$$
S^{\Phi \Phi h^{(4)}}\left(\Phi, h^{(2)}, h^{(4)}\right)=S_{0}(\Phi)+S_{1}\left(\Phi, h^{(2)}\right)+S_{1}\left(\Phi, h^{(4)}\right),
$$


where $S_{0}(\Phi), S_{1}\left(\Phi, h^{(2)}\right)$ are defined in (1)-(3) and

$$
S_{1}\left(\Phi, h^{(4)}\right)=\frac{1}{4} \int d^{d} x h^{(4) \mu \nu \alpha \beta}\left[\partial_{\mu} \partial_{\nu} \Phi \partial_{\alpha} \partial_{\beta} \Phi-\eta_{\mu \nu} \partial_{\alpha} \partial^{\gamma} \Phi \partial_{\beta} \partial_{\gamma} \Phi\right]
$$

From now on to avoid cumbersome notation and overlapping with symmetrization brackets we reserve notation $h$ and varepsilon for gauge field for spin four $h(4)$ and corresponding gauge parameter $\varepsilon^{(3)}$ except cases when we do not explicitly write out indices. In the case of other spin (rank) fields and parameters, we use these letters with exact indication of rank.

The action (1)is invariant with respect to the gauge transformations of the spin four field with an additional spin two field gauge transformation inspired by the second divergence of the spin four gauge parameter ${ }^{1}$

$$
\begin{aligned}
\delta_{1} \Phi(x) & =\varepsilon^{\mu \nu \lambda}(x) \partial_{\mu} \partial_{\nu} \partial_{\lambda} \Phi(x) \\
\delta_{0} h^{\mu \nu \lambda \rho} & =\partial^{(\mu} \varepsilon^{\nu \lambda \rho)}=\partial^{\mu} \varepsilon^{\nu \lambda \rho}+\partial^{v} \varepsilon^{\mu \lambda \rho}+\partial^{\lambda} \varepsilon^{\mu \nu \rho}+\partial^{\rho} \varepsilon^{\mu \nu \lambda} \\
\delta_{0} h_{(2)}^{\mu \nu} & =\partial^{(\mu} \epsilon^{\nu)} \\
\epsilon^{\nu} & =\partial_{\alpha} \partial_{\beta} \varepsilon^{\nu \alpha \beta}
\end{aligned}
$$

For further simplification in calculation of quartic terms in spin four case we will use physical traceless and transfer gauge for our external spin four field:

$$
\begin{aligned}
\partial_{\mu} h^{\mu \nu \lambda \rho} & =0 \\
h_{\mu}^{\mu \lambda \rho} & =0
\end{aligned}
$$

which leads to the corresponding restrictions on already traceless spin four gauge parameter:

$$
\begin{aligned}
\partial_{\alpha} \varepsilon^{\alpha \beta \gamma} & =0 \\
\partial_{\mu} \partial^{\mu} \varepsilon^{\alpha \beta \gamma} & =\square \varepsilon^{\alpha \beta \gamma}=0
\end{aligned}
$$

Note that because in our gauge the gauge parameter is transfer, we should get decoupling of spin two mode from spin four due to degeneration of the additional gauge transformation (5). Another convention is that from now on we will admit integration everywhere where it is necessary. So we work with a Lagrangian as with action and therefore we will neglect all d dimensional space-time total derivatives when making a partial integration.

\section{variation of cubic term}

So we arrive to the following simplified task: Starting from a single cubic term due to (7)-(10) ${ }^{2}$

$$
L_{1} \sim h^{\mu \nu \lambda \rho} \partial_{\mu} \partial_{\nu} \Phi \partial_{\lambda} \partial_{\rho} \Phi
$$

and using known variation:

$$
\begin{aligned}
\delta_{1} \Phi & =\varepsilon^{\alpha \beta \gamma} \partial_{\alpha} \partial_{\beta} \partial_{\gamma} \Phi \\
\delta_{0} h^{\mu \nu \lambda \rho} & =\partial^{(\mu} \varepsilon^{\nu \lambda \rho)}
\end{aligned}
$$

\footnotetext{
${ }^{1}$ Note that the spin two part of our action continues to be invariant in respect of usual linearized reparametrization

${ }^{2}$ As usual in our articles we widely use Lagrangian instead of Action performing Noether procedure admitting possibility for partial integration
} 
we try to solve functional equation:

$$
\delta_{1} L_{1}\left(\Phi, h^{(4)}\right)+\delta_{0} L_{2}\left(\Phi, h^{(4)}\right)=0
$$

and construct unknown quartic interaction and first order gauge variation of spin four field $\delta_{1} h^{\mu \nu \lambda \rho}$. Duing that and taking into account that according to (7) and (9) $\alpha, \beta, \gamma$ derivatives commute with $\varepsilon$ and $\mu, v, \lambda, \rho$ derivatives commute with $h$ and after long manipulations and multiple partial integrations we arrive to the following important variation:

$$
\begin{aligned}
\delta_{1}\left(h^{\mu \nu \lambda \rho} \partial_{\mu} \partial_{\nu} \Phi \partial_{\lambda} \partial_{\rho} \Phi\right) & =\frac{1}{3} \delta_{1}\left(h^{\mu \nu \lambda \rho} J_{\mu \nu \lambda \rho}^{(4)}\right)=\delta_{1} h^{\mu \nu \lambda \rho} \partial_{\mu} \partial_{\nu} \Phi \partial_{\lambda} \partial_{\rho} \Phi \\
& +\frac{1}{50}\left[\varepsilon^{\mu(\alpha \beta} \partial_{\mu} h^{\gamma \nu \lambda \rho)}-\partial_{\mu} \varepsilon^{(\alpha \beta \gamma} h^{\nu \lambda \rho) \mu}\right] J_{\nu \lambda \rho \alpha \beta \gamma}^{(6)} \\
& +\frac{1}{5}\left[\partial_{\alpha} \varepsilon^{\mu \nu(\beta} \partial_{\mu} \partial_{\nu} h^{\gamma \lambda \rho) \alpha}-\partial_{\mu} \partial_{\nu} \varepsilon^{\alpha(\beta \gamma} \partial_{\alpha} h^{\lambda \rho) \mu \nu}\right] J_{\lambda \rho \beta \gamma}^{(4)} \\
& +\frac{2}{15}\left[\partial_{\alpha} \partial_{\beta} \partial_{\gamma} \varepsilon^{(\mu \nu \lambda} h^{\rho) \alpha \beta \gamma}-\varepsilon^{\alpha \beta \gamma} \partial_{\alpha} \partial_{\beta} \partial_{\gamma} h^{\mu \nu \lambda \rho}\right] J_{\mu \nu \lambda \rho}^{(4)} \\
& +\frac{1}{5}\left[\partial_{\mu} \partial_{\nu} \varepsilon^{\alpha \beta \gamma} \partial_{\alpha} \partial_{\beta} \partial_{\gamma} h^{\mu \nu \lambda \rho}-\partial_{\mu} \partial_{\nu} \partial_{\gamma} \varepsilon^{\alpha \beta(\lambda} \partial_{\alpha} \partial_{\beta} h^{\rho) \mu \nu \gamma}\right] J_{\lambda \rho}^{(2)} \\
& +\frac{1}{5} \partial_{\mu} \partial_{\nu} \partial_{\lambda} \partial_{\rho} \varepsilon^{\alpha \beta \gamma} \partial_{\alpha} h^{\mu \nu \lambda \rho} J_{\beta \gamma}^{(2)}
\end{aligned}
$$

Here $J^{(6)}, J^{(4)}, J^{(2)}$ are symmetrized currents:

$$
\begin{aligned}
J_{\nu \lambda \rho \alpha \beta \gamma}^{(6)} & =\partial_{(\nu} \partial_{\lambda} \partial_{\rho} \Phi \partial_{\alpha} \partial_{\beta} \partial_{\gamma)} \Phi=\partial_{\nu} \partial_{(\lambda} \partial_{\rho} \Phi \partial_{\alpha} \partial_{\beta} \partial_{\gamma)} \Phi, & & 10 \text { terms }=\frac{6 !}{3 ! 3 ! 2}=\frac{5 !}{3 ! 2 !}, \\
J_{\mu \nu \lambda \rho}^{(4)} & =\partial_{(\mu} \partial_{\nu} \Phi \partial_{\lambda} \partial_{\rho)} \Phi=\partial_{\mu} \partial_{(\nu} \Phi \partial_{\lambda} \partial_{\rho)} \Phi, & & 3 \text { terms }=\frac{4 !}{2 ! 2 ! 2}=\frac{3 !}{2 ! 1 !}, \\
J_{\mu \nu}^{(2)} & =\partial_{\mu} \Phi \partial_{\nu} \Phi, & & 1 \text { term }
\end{aligned}
$$

From (15) we see several differences from spin two case:

- From second line of (15) follows that we cannot integrate Noether's equation without introduction of the cubic interaction with a gauge field of spin 6 coupled to the spin 6 current:

$$
h_{(6)}^{v \lambda \rho \alpha \beta \gamma} J_{v \lambda \rho \alpha \beta \gamma}^{(6)}
$$

- From third and fourth lines we see that $J^{(4)}$ terms arose with different weight $\frac{1}{5}$ and $\frac{2}{15}$. But we will see below that they should come with same weight to complete integration for interaction terms.

- In last two lines we have three unwanted $J^{(2)}$ terms. We should discover way to get rid of them.

To remove these three obstructions we note that there are several connections between our parts in (15) leading to a redefinition of the initial cubic interactions. In another words we can modify our initial interaction with higher spin currents adding gradients of lower spin currents with some coefficients:

$$
\begin{aligned}
J_{\alpha \beta \mu \nu \lambda \rho}^{(6)} & =>J_{\alpha \beta \mu \nu \lambda \rho}^{(6)}+A \partial_{(\alpha} \partial_{\beta} J_{\mu \nu \lambda \rho)}^{(4)}+B \partial_{(\alpha} \partial_{\beta} \partial_{\mu} \partial_{\nu} J_{\lambda \rho)}^{(2)} \\
J_{\mu \nu \lambda \rho}^{(4)} & =>J_{\mu \nu \lambda \rho}^{(4)}+C \partial_{(\mu} \partial_{\nu} J_{\lambda \rho)}^{(2)}
\end{aligned}
$$


And it works! Hiding all details of derivations we present final variation we obtained by tuning procedure (20) instead of (15):

$$
\begin{aligned}
\delta_{1}\left(h^{\mu \nu \lambda \rho} \partial_{\mu} \partial_{\nu} \Phi \partial_{\lambda} \partial_{\rho} \Phi\right) & =\frac{1}{3} \delta_{1}\left(h^{\mu \nu \lambda \rho} J_{\mu \nu \lambda \rho}^{(4)}\right)=\delta_{1} h^{\mu \nu \lambda \rho} \partial_{\mu} \partial_{\nu} \Phi \partial_{\lambda} \partial_{\rho} \Phi \\
& +\frac{1}{50}\left[\varepsilon^{\mu(\alpha \beta} \partial_{\mu} h^{\gamma \nu \lambda \rho)}-\partial_{\mu} \varepsilon^{(\alpha \beta \gamma} h^{\nu \lambda \rho) \mu}\right] \tilde{J}_{\nu \lambda \rho \alpha \beta \gamma}^{(6)} \\
& +\frac{1}{6}\left[\partial_{\alpha} \varepsilon^{\mu \nu(\beta} \partial_{\mu} \partial_{\nu} h^{\gamma \lambda \rho) \alpha}-\partial_{\mu} \partial_{\nu} \varepsilon^{\alpha(\beta \gamma} \partial_{\alpha} h^{\lambda \rho) \mu \nu}\right] J_{\lambda \rho \beta \gamma}^{(4)} \\
& +\frac{1}{6}\left[\partial_{\alpha} \partial_{\beta} \partial_{\gamma} \varepsilon^{(\mu \nu \lambda} h^{\rho) \alpha \beta \gamma}-\varepsilon^{\alpha \beta \gamma} \partial_{\alpha} \partial_{\beta} \partial_{\gamma} h^{\mu \nu \lambda \rho}\right] J_{\mu \nu \lambda \rho}^{(4)}
\end{aligned}
$$

where modified $\tilde{J}^{(6)}$ is

$$
\tilde{J}_{\nu \lambda \rho \alpha \beta \gamma}^{(6)}=J_{\nu \lambda \rho \alpha \beta \gamma}^{(6)}+\frac{1}{9} \partial_{(\alpha} \partial_{\beta} J_{\gamma \nu \lambda \rho)}^{(4)}+\frac{1}{3} \partial_{(\nu} \partial_{\lambda} \partial_{\rho} \partial_{\alpha} J_{\beta \gamma)}^{(2)}
$$

Supplemented by traceless Stueckelberg like transformation of the spin two gauge field from linear coupling with $J^{(2)}$ current:

$$
\delta_{1} h_{(2)}^{\beta \gamma} \sim \partial_{\mu} \partial_{\nu} \partial_{\lambda} \partial_{\rho} \varepsilon^{\alpha \beta \gamma} \partial_{\alpha} h^{\mu \nu \lambda \rho}
$$

\section{Integration and interaction}

Now we can start to integrate the last three lines of expression (22). Doing that and hiding again long but straightforward calculations we finally obtain quartic interactions:

$$
\begin{gathered}
S_{2}\left(\Phi, h^{(4)}\right)=\int d^{d} x\left\{\frac{1}{10} h_{\mu}^{\alpha \beta \gamma} h^{\nu \lambda \rho \mu} \tilde{J}_{\nu \lambda \rho \alpha \beta \gamma}^{(6)}\right. \\
-\frac{2}{3} h_{\mu}^{\alpha \beta \gamma} \partial_{\alpha} \partial_{\beta} h^{\mu \nu \lambda \rho} J_{\nu \lambda \rho \gamma}^{(4)}+\frac{1}{2} \partial_{\nu} h_{\mu}^{\alpha \beta \gamma} \partial_{\alpha} h^{\mu \nu \lambda \rho} J_{\lambda \rho \beta \gamma}^{(4)}-\frac{1}{4} \partial^{\alpha} h_{\mu \nu}^{\beta \gamma} \partial_{\alpha} h^{\mu \nu \lambda \rho} J_{\lambda \rho \beta \gamma}^{(4)} \\
\left.-\partial^{\beta} h_{\mu \nu}^{\alpha \gamma} \partial_{\alpha} h^{\mu \nu \lambda \rho} J_{\lambda \rho \beta \gamma}^{(4)}+\frac{1}{3} \partial^{\beta} h_{\mu \nu \lambda}^{\gamma} \partial^{\alpha} h^{\mu \nu \lambda \rho} J_{\rho \alpha \beta \gamma}^{(4)}-\frac{1}{4} h_{\mu \nu}^{\beta \gamma} h^{\lambda \rho \mu \nu} \square J_{\lambda \rho \beta \gamma}^{(4)}\right\}
\end{gathered}
$$

and linear on spin four gauge field transformations fixed by Noether's procedure:

$\delta_{1} h_{(6)}^{\mu \nu \lambda \alpha \beta \gamma}=\varepsilon^{\rho(\alpha \beta} \partial_{\rho} h^{\gamma \mu \nu \lambda)}+\partial^{(\alpha} \varepsilon_{\rho}^{\beta \gamma} h^{\mu \nu \lambda) \rho}$

$\delta_{1} h^{\mu \nu \lambda \rho} \sim \varepsilon^{\alpha \beta \gamma} \partial_{\alpha} \partial_{\beta} \partial_{\gamma} h^{\mu \nu \lambda \rho}+\partial^{(\mu} \varepsilon_{\gamma}^{|\alpha \beta|} \partial_{\alpha} \partial_{\beta} h^{\nu \lambda \rho) \gamma}+\partial^{(\mu} \partial^{v} \varepsilon_{\beta \gamma}^{|\alpha|} \partial_{\alpha} h^{\lambda \rho) \beta \gamma}+\partial^{(\mu} \partial^{v} \partial^{\lambda} \varepsilon_{\alpha \beta \gamma} h^{\rho) \alpha \beta \gamma}$

$\delta_{1} h_{(2)}^{\beta \gamma} \sim \partial_{\mu} \partial_{\nu} \partial_{\lambda} \partial_{\rho} \varepsilon^{\alpha \beta \gamma} \partial_{\alpha} h^{\mu \nu \lambda \rho}$

\section{Commutator of $\delta_{1}$ transformations for spin four}

In this section we investigate the structure of linear in gauge field transformation (27)

$$
\begin{gathered}
\delta_{1}^{(\varepsilon)} h_{\mu \nu \lambda \rho}=\varepsilon^{\alpha \beta \gamma} \partial_{\alpha} \partial_{\beta} \partial_{\gamma} h_{\mu \nu \lambda \rho}+\partial_{(\mu} \varepsilon^{\alpha \beta \gamma} \partial_{\mid \alpha} \partial_{\beta} h_{\gamma \mid \nu \lambda \rho)}+\partial_{(\mu} \partial_{\nu} \varepsilon^{\alpha \beta \gamma} \partial_{\mid \alpha} h_{\beta \gamma \mid \lambda \rho)} \\
+\partial_{(\mu} \partial_{\nu} \partial_{\lambda} \varepsilon^{\alpha \beta \gamma} h_{\rho) \alpha \beta \gamma}
\end{gathered}
$$


The structure of this expression is similar to linear transformation obtained in [29] where nonlinear curvature for spin three case is considered. Following the ideas of spin three case after some straightforward manipulations with derivatives we can rewrite (1) in the following form:

$$
\begin{aligned}
\delta_{1}^{(\varepsilon)} h_{\mu \nu \lambda \rho} & =\varepsilon^{\alpha \beta \gamma} \Gamma_{\alpha \beta \gamma ; \mu \nu \lambda \rho}(h)+\partial_{(\mu} \Lambda_{\nu \lambda \rho)}(\varepsilon, h) \\
\Lambda_{\nu \lambda \rho}(\varepsilon, h) & =\varepsilon^{\alpha \beta \gamma} \partial_{\alpha} \partial_{\beta} h_{\gamma \nu \lambda \rho}+\frac{1}{2}\left[\partial_{(\nu} \varepsilon^{\alpha \beta \gamma} \partial_{\mid \alpha} h_{\beta \gamma \mid \lambda \rho)}-\varepsilon^{\alpha \beta \gamma} \partial_{(\nu} \partial_{\mid \alpha} h_{\beta \gamma \mid \lambda \rho)}\right] \\
& +\frac{1}{3}\left[\partial_{(\nu} \partial_{\lambda} \varepsilon^{\alpha \beta \gamma} h_{\rho) \alpha \beta \gamma}+\varepsilon^{\alpha \beta \gamma} \partial_{(\nu} \partial_{\lambda} h_{\rho) \alpha \beta \gamma}-\frac{1}{2} \partial_{(\nu} \varepsilon^{\alpha \beta \gamma} \partial_{\lambda} h_{\rho) \alpha \beta \gamma}\right]
\end{aligned}
$$

where

$$
\begin{aligned}
\Gamma_{\alpha \beta \gamma ; \mu \nu \lambda \rho}^{(3)}(h) & =\partial_{\alpha} \partial_{\beta} \partial_{\gamma} h_{\mu \nu \lambda \rho}-\frac{1}{3} \partial_{<\alpha} \partial_{\beta} \partial_{(\mu} h_{\nu \lambda \rho) \gamma>}+\frac{1}{3} \partial_{<\alpha} \partial_{(\mu} \partial_{\nu} h_{\lambda \rho) \beta \gamma>} \\
& -\partial_{(\mu} \partial_{\nu} \partial_{\lambda} h_{\rho) \alpha \beta \gamma}
\end{aligned}
$$

is the third for spin four gauge field (last before Curvature) Christoffel Symbol in deWit-Freedman hierarchy of connections defined in [32]

The key point of the splitting (2) is the simple form of zero order on field gauge transformation of connection (3):

$$
\delta_{0}^{(\varepsilon)} \Gamma_{\alpha \beta \gamma ; \mu \nu \lambda \rho}^{(3)}(h)=-4 \partial_{\mu} \partial_{\nu} \partial_{\lambda} \partial_{\rho} \varepsilon_{\alpha \beta \gamma}
$$

So we see that expressions (1)-(5) is really looks like higher spin generalization of the gauge transformation (16) (Lie derivative) and usual Christoffel symbol for linearized gravity ${ }^{3}$

$$
\begin{aligned}
\delta_{1}^{(\varepsilon)} h_{\mu \nu} & =\mathfrak{L}_{\varepsilon^{\lambda}} h_{\mu \nu}=\varepsilon^{\alpha} \Gamma_{\alpha ; \mu \nu}^{(1)}+\partial_{(\mu}\left(\varepsilon^{\alpha} h_{\nu) \alpha}\right) \\
\Gamma_{\alpha ; \mu \nu}^{(1)} & =\partial_{\alpha} h_{\mu \nu}-\partial_{(\mu} h_{\nu) \alpha} \\
\delta_{0}^{(\varepsilon)} \Gamma_{\alpha ; \mu \nu}^{(1)}(h) & =-2 \partial_{\mu} \partial_{\nu} \varepsilon_{\alpha}
\end{aligned}
$$

Using representation (2) and transformation rule (5) we can derive the following expression for commutator:

$$
\begin{aligned}
{\left[\delta_{1}^{(\omega)}, \delta_{1}^{(\varepsilon)}\right] h_{\mu \nu \lambda \rho} } & =\varepsilon^{\alpha \beta \gamma} \Gamma_{\alpha \beta \gamma ; \mu \nu \lambda \rho}^{(3)}\left(\delta_{1}^{(\omega)} h\right)-4 \varepsilon^{\alpha \beta \gamma} \partial_{\mu} \partial_{\nu} \partial_{\lambda} \partial_{\rho} \Lambda_{\alpha \beta \gamma}(\omega, h) \\
& +\partial_{(\mu} \Lambda_{\nu \lambda \rho)}\left(\varepsilon, \delta_{1}^{(\omega)} h\right)-(\varepsilon \leftrightarrow \omega)
\end{aligned}
$$

Then taking int account that all symmetrized full gradients in r.h.s we can drop as a trivial $\delta_{0}$ contribution from composite symmetric third rank gauge parameter linear in gauge field, we can first of all drop second line in (9). Then we can put four $\mu, v, \lambda, \rho$, derivatives in second term of first line from $\Lambda_{\alpha \beta \gamma}$ to parameter $\varepsilon^{\alpha \beta \gamma}$ and integrate using formula (5) and came to the following expression

$$
\left[\delta_{1}^{(\omega)}, \delta_{1}^{(\varepsilon)}\right] h_{\mu \nu \lambda \rho} \sim \varepsilon^{\alpha \beta \gamma} \Gamma_{\alpha \beta \gamma ; \mu \nu \lambda \rho}^{(3)}\left(\delta_{1}^{(\omega)} h\right)+\Gamma_{\alpha \beta \gamma ; \mu \nu \lambda \rho}^{(3)}(h) \delta_{0}^{(\omega)} \Lambda^{\alpha \beta \gamma}(\varepsilon, h)-(\varepsilon \leftrightarrow \omega)
$$

\footnotetext{
${ }^{3}$ Note that most common definition of Christoffel symbol $\Gamma_{\mu \nu}^{\beta}(g)=\frac{1}{2} g^{\beta \alpha}\left(\partial_{(\mu} g_{\nu) \alpha}-\partial_{\alpha} g_{\mu \nu}\right)$ for general metric $g_{\mu \nu}$ relates with our definition after linearization in the flat background in the following way $\Gamma_{\mu \nu}^{\beta}\left(\eta_{\mu \nu}+h_{\mu \nu}\right)=$ $-\frac{1}{2} \eta^{\beta \alpha} \Gamma_{\alpha ; \mu \nu}^{(1)}(h)$
} 
where $\sim$ means an equality up to any $\delta_{0}$ variations with composed field dependent parameter described above or delta zero variation with usual parameter $\varepsilon$ or $\omega$ from any second order on gauge field tensor. At this point it is worth to note that considering perturbative on linearized gauge field deformation of the initial gauge transformation regulated by Neether's procedure

$$
\delta^{(\epsilon)} h_{\mu \nu \lambda \rho}=\left(\delta_{0}^{(\epsilon)}+\delta_{1}^{(\epsilon)}+\delta_{2}^{(\epsilon)}+\ldots\right) h_{\mu \nu \lambda \rho},
$$

for commutator on the linear level on gauge field we obtain:

$$
\left\{\left[\delta^{(\omega)}, \delta^{(\epsilon)}\right] h_{\mu \nu \lambda \rho}\right\}_{1}=\left(\left[\delta_{1}^{(\omega)}, \delta_{1}^{(\epsilon)}\right]+\delta_{0}^{(\omega)} \delta_{2}^{(\varepsilon)}-\delta_{0}^{(\varepsilon)} \delta_{2}^{(\omega)}\right) h_{\mu \nu \lambda \rho}
$$

So we see that we can factorize in right hand side of our commutator of the first order gauge transformation two type of trivial terms:

- Symmetrized full derivatives from composed gauge parameter linear in gage fields $\partial_{(\mu} \tilde{\Lambda}_{\nu \lambda \rho)}(\varepsilon, h)-$ $(\varepsilon \leftrightarrow \omega)$.

- The terms which can be classified as a second part of (12):

$\delta_{0}^{(\omega)} \delta_{2}^{(\varepsilon)} h_{\mu \nu \lambda \rho}-(\varepsilon \leftrightarrow \omega)$ and we can throw them out also to understand algebra of two $\delta_{1}$ transformations.

Now following this simple methodology we can present final result for commutator hiding long and tedious calculation for next journal's publication:

$$
\begin{aligned}
& {\left[\delta_{1}^{(\omega)}, \delta_{1}^{(\varepsilon)}\right] h_{\mu \nu \lambda \rho} \sim\left[\varepsilon^{\delta \sigma \eta} \partial_{\delta} \partial_{\sigma} \partial_{\eta} \omega^{\alpha \beta \gamma}+T^{\alpha \beta \gamma}(\partial, \varepsilon, \omega)\right] \Gamma_{\alpha \beta \gamma ; \mu \nu \lambda \rho}^{(3)}(h)} \\
& +3 \varepsilon^{\delta \sigma \eta} \partial_{\delta} \partial_{\sigma} \omega^{\alpha \beta \gamma} R_{\eta \alpha \beta \gamma ; \mu \nu \lambda \rho}^{(4)}(h)+\frac{9}{20} \varepsilon_{\delta}^{\sigma \eta} \partial^{[\delta} \omega^{\alpha] \beta \gamma} \partial_{(\sigma} R_{\eta \alpha \beta \gamma) ; \mu \nu \lambda \rho}^{(4)}(h) \\
& +[\operatorname{Rem}]_{\mu \nu \lambda \rho}(\varepsilon, \omega, h)-(\varepsilon \leftrightarrow \omega)
\end{aligned}
$$

where:

$$
\begin{aligned}
T^{\alpha \beta \gamma}(\partial, \varepsilon, \omega) & =\frac{1}{4} \partial^{(\alpha} \partial^{\beta} \varepsilon^{\delta \sigma \eta} \delta_{0}^{(\omega)} h_{\delta \sigma \eta}^{\gamma)}-\frac{5}{48} \partial^{(\alpha} \varepsilon^{\delta \sigma \eta} \partial^{\beta} \delta_{0}^{(\omega)} h_{\delta \sigma \eta}^{\gamma)}+\frac{7}{16} \partial^{(\alpha} \varepsilon^{\delta \sigma \eta} \partial_{\delta} \delta_{0}^{(\omega)} h_{\sigma \eta}^{\beta \gamma)} \\
& -\frac{1}{16} \partial^{\delta} \varepsilon^{\sigma \eta(\alpha} \partial^{\beta} \delta_{0}^{(\omega)} h_{\delta \sigma \eta}^{\gamma)}+\frac{1}{16} \partial^{\delta} \varepsilon^{\sigma \eta(\alpha} \partial_{\delta} \delta_{0}^{(\omega)} h_{\sigma \eta}^{\beta \gamma)}
\end{aligned}
$$

$R_{\eta \alpha \beta \gamma ; \mu \nu \lambda \rho}^{(4)}(h)$ here is de Wit-Freedman higher spin curvature [32]:

$$
\begin{aligned}
R_{\eta \alpha \beta \gamma ; \mu \nu \lambda \rho}^{(4)}\left(h^{(4)}\right) & =\partial_{\eta} \partial_{\alpha} \partial_{\beta} \partial_{\gamma} h_{\mu \nu \lambda \rho}^{(4)}-\frac{1}{4} \partial_{<\eta} \partial_{\alpha} \partial_{\beta} \partial_{(\mu} h_{\nu \lambda \rho) \gamma>}^{(4)} \\
& +\frac{1}{6} \partial_{<\eta} \partial_{\alpha} \partial_{(\mu} \partial_{\nu} h_{\lambda \rho) \beta \gamma>}^{(4)}-\frac{1}{4} \partial_{<\eta} \partial_{(\mu} \partial_{\nu} \partial_{\lambda} h_{\rho) \alpha \beta \gamma>}^{(4)}+\partial_{\mu} \partial_{\nu} \partial_{\lambda} \partial_{\rho} h_{\eta \alpha \beta \gamma}^{(4)}
\end{aligned}
$$

and the Remainder $[\operatorname{Rem}]_{\mu \nu \lambda \rho}(\varepsilon, \omega, h)$ is Contributions from Gauge Transformation of Spin four Field with Mixed Symmetry Tensor Parameter.

So we arrived to the statement that all irrelevant contributions to the r.h.s. of our commutator is an action of the different mixed symmetry higher spin gauge transformation acting on our spin four gauge field. Therefore when our quartic vertex is constructed from special fields including scalars this contribution could be zero due to the commutativity of flat space derivatives working with scalar field and we can solve Noether's procedure in the framework of a set of symmetric tensor fields with spin $2 \leq s \leq 6$. 


\section{Outlook}

Here we just list possible tasks for future investigation. So What can be done

- "Degauging " and "Off-Shelling" of our quartic interactions constructed for special constrained gauge fields

- Generalization for spin higher than four and scalars.

- Inclusion in the game of the generalized Weyl invariance considered in [27] and [28].

- What's cooking in the case of different spins?

- More special cases?

So we stop here with the hope to continue these investigations.

\section{References}

[1] M. A. Vasiliev, "Consistent equation for interacting gauge fields of all spins in $(3+1)$ dimensions.", Phys. Lett. B 243 (1990) 378-382. M. A. Vasiliev, " Nonlinear equations for symmetric massless higher spin fields in $(A) d S_{d}$." Phys. Lett. B 567 (2003) 139-151, arXiv:hep-th/0304049.

[2] M. A. Vasiliev, "V L Ginzburg and higher-spin fields," Phys. Usp. 54, 641 (2011) [Usp. Fiz. Nauk 181, 665 (2011)].

[3] A. Sagnotti, "Notes on Strings and Higher Spins," J. Phys. A 46 (2013) 214006 [arXiv:1112.4285 [hep-th]].

[4] C. Sleight, "Metric-like Methods in Higher Spin Holography," PoS Modave 2016 (2017) 003 [arXiv:1701.08360 [hep-th]].

[5] S. Giombi, I. R. Klebanov and Z. M. Tan, "The ABC of Higher-Spin AdS/CFT," Universe 4 (2018) no.1, 18 doi:10.3390/universe4010018 [arXiv:1608.07611 [hep-th]].

[6] S. Giombi, "Higher Spin — CFT Duality,” arXiv:1607.02967 [hep-th].

[7] R. Rahman and M. Taronna, "From Higher Spins to Strings: A Primer," arXiv:1512.07932 [hep-th].

[8] A. K. H. Bengtsson, I. Bengtsson and L. Brink, "Cubic Interaction Terms For Arbitrary Spin," Nucl. Phys. B 227 (1983) 31. "Cubic Interaction Terms For Arbitrarily Extended Supermultiplets,” Nucl. Phys. B 227 (1983) 41.

[9] R. R. Metsaev, "Cubic interaction vertices for massive and massless higher spin fields," Nucl. Phys. B 759 (2006) 147 [arXiv:hep-th/0512342]; R. R. Metsaev, "Cubic interaction vertices for fermionic and bosonic arbitrary spin fields," arXiv:0712.3526 [hep-th]. 
[10] R. R. Metsaev, "Cubic interaction vertices for $\mathrm{N}=1$ arbitrary spin massless supermultiplets in flat space," arXiv:1905.11357 [hep-th]; "Cubic interaction vertices for massive/massless continuous-spin fields and arbitrary spin fields," JHEP 1812 (2018) 055 doi:10.1007/JHEP12(2018)055 [arXiv:1809.09075 [hep-th]].

[11] F. A. Berends, G. J. H. Burgers and H. Van Dam, "On Spin Three Selfinteractions," Z. Phys. C 24 (1984) 247; F. A. Berends, G. J. H. Burgers and H. van Dam, "On The Theoretical Problems In Constructing Interactions Involving Higher Spin Massless Particles,” Nucl. Phys. B 260 (1985) 295.; F. A. Berends, G. J. H. Burgers and H. van Dam, "Explicit Construction Of Conserved Currents For Massless Fields Of Arbitrary Spin,” Nucl. Phys. B 271 (1986) 429;

[12] E. S. Fradkin and M. A. Vasiliev, "On The Gravitational Interaction Of Massless Higher Spin Fields," Phys. Lett. B 189 (1987) 89; E. S. Fradkin and M. A. Vasiliev, "Cubic Interaction In Extended Theories Of Massless Higher Spin Fields,” Nucl. Phys. B 291 (1987) 141.

[13] R. Manvelyan, K. Mkrtchyan and W. Rühl, "Off-shell construction of some trilinear higher spin gauge field interactions,” Nucl. Phys. B 826 (2010) 1; arXiv: 0903.0243.

[14] X. Bekaert, E. Joung and J. Mourad, “On higher spin interactions with matter," JHEP 0905 (2009) 126; arXiv:0903.3338.

[15] R. Manvelyan, K. Mkrtchyan and W. Rühl, "General trilinear interaction for arbitrary even higher spin gauge fields,” Nucl. Phys. B 836 (2010) 204; arXiv: 1003.2877.

[16] R. Manvelyan, K. Mkrtchyan and W. Rühl, "Direct Construction of A Cubic Selfinteraction for Higher Spin gauge Fields,” Nucl. Phys. B 844 (2011) 348; arXiv: 1002 . 1358.

[17] A. Sagnotti and M. Taronna, "String Lessons for Higher-Spin Interactions," Nucl. Phys. B 842 (2011) 299; arXiv: 1006.5242.

[18] R. Manvelyan, K. Mkrtchyan and W. Rühl, "A Generating function for the cubic interactions of higher spin fields,” Phys. Lett. B 696 (2011) 410; arXiv: 1009. 1054.

[19] Yu.M. Zinoviev, "Spin 3 cubic vertices in a frame-like formalism." JHEP 1008:084,2010; arXiv:1007.0158 [hep-th]

[20] M. A. Vasiliev, "Cubic Vertices for Symmetric Higher-Spin Gauge Fields in $(A) d S_{d}$," Nucl. Phys. B 862 (2012) 341 doi:10.1016/j.nuclphysb.2012.04.012 [arXiv:1108.5921 [hep-th]].

[21] P. Dempster and M. Tsulaia, "On the Structure of Quartic Vertices for Massless Higher Spin Fields on Minkowski Background,” Nucl. Phys. B 865 (2012) 353; arXiv: 1203.5597.

[22] A. K. H. Bengtsson, "Investigations into Light-front Quartic Interactions for Massless Fields (I): Non-constructibility of Higher Spin Quartic Amplitudes,” JHEP 1612 (2016) 134; arXiv: 1607.06659.

[23] M. Taronna, "On the Non-Local Obstruction to Interacting Higher Spins in Flat Space," JHEP 1705 (2017) 026; arXiv: 1701.05772. 
[24] R. Roiban and A. A. Tseytlin, "On four-point interactions in massless higher spin theory in flat space,” JHEP 1704 (2017) 139; arXiv: 1701.05773.

[25] S. Fredenhagen, O. Krüger and K. Mkrtchyan, "Vertex-Constraints in 3D Higher Spin Theories," arXiv:1905.00093 [hep-th].

[26] S. Fredenhagen, O. Krüger and K. Mkrtchyan, "Constraints for Three-Dimensional HigherSpin Interactions and Conformal Correlators," arXiv:1812.10462 [hep-th].

[27] R. Manvelyan and W. Rühl, "Conformal coupling of higher spin gauge fields to a scalar field in $A d S_{4}$ and generalized Weyl invariance", Phys. Lett. B 593 (2004) 253 [arXiv:hep-th/0403241];

[28] R. Manvelyan and K. Mkrtchyan, "Conformal invariant interaction of a scalar field with the higher spin field in AdS(D)," Mod. Phys. Lett. A 25 (2010) 1333 [arXiv:0903.0058 [hep-th]].

[29] R. Manvelyan, K. Mkrtchyan, W. Rühl and M. Tovmasyan, "On Nonlinear Higher Spin Curvature,” Phys. Lett. B 699 (2011), 187-191 doi:10.1016/j.physletb.2011.03.069 [arXiv:1102.0306 [hep-th]].

[30] R. Manvelyan and W. Rühl, "Generalized Curvature and Ricci Tensors for a Higher Spin Potential and the Trace Anomaly in External Higher Spin Fields in AdS(4) Space," Nucl. Phys. B 796 (2008), 457-470 doi:10.1016/j.nuclphysb.2007.11.040 [arXiv:0710.0952 [hepth]].

[31] R. Manvelyan and W. Rühl, "The Generalized curvature and Christoffel symbols for a higher spin potential in AdS(d+1) space," Nucl. Phys. B 797 (2008), 371-394 doi:10.1016/j.nuclphysb.2007.10.012 [arXiv:0705.3528 [hep-th]].

[32] B. de Wit and D. Z. Freedman, "Systematics of Higher Spin Gauge Fields," Phys. Rev. D 21 (1980), 358 doi:10.1103/PhysRevD.21.358 